\title{
Multidisciplinary treatment for macrometastatic osteogenic sarcoma
}

\author{
NORMAN JAFFE, DEMETRIUS TRAGGIS, J ROBERT CASSADY, ROBERT M FILLER, \\ HUGH WATTS, EMIL FREI
}

British Medical fournal, 1976, 2, 1039-1041

\begin{abstract}
Summary
Using a co-ordinated multidisciplinary approach with surgery, radiation therapy, and chemotherapy, 14 out of 21 patients with metastases from osteogenic sarcoma were rendered free of disease for over two to over 18 months. Most patients had pulmonary metastases, two had bony metastases, and one had metastases in the iliac nodes. As part of this multidisciplinary approach weekly high-dose methotrexate was given and caused tumour regression in seven out of 15 patients. After all clinical evidence of disease had been removed high-dose methotrexate was administered every two to three weeks as maintenance treatment. To assess the efficacy of treatment the results were compared with those in a historical control group of 82 consecutive patients who developed pulmonary or other metastases. The results in the study group were significantly better. This experience may be similar to that in Wilms's tumour, where actinomycin D has increased the cure rate when administered as adjuvant therapy after treatment of localised or overt metastatic disease.
\end{abstract}

\section{Introduction}

The effectiveness of the vincristine, high-dose methotrexate, citrovorum factor "rescue" programme (V-MTX-CF) in osteogenic sarcoma is established. This regimen, appropriately applied, will (a) affect tumour regression of primary lesions in preparation for subamputative surgery, ${ }^{12}$ (b) control micrometastases (adjuvant chemotherapy), ${ }^{3-6}$ and (c) affect tumour regression of overt metastatic disease. ${ }^{2}{ }^{78} \mathrm{We}$ report here the integration of V-MTX-CF in a multidisciplinary treatment programme for overt metastatic osteogenic sarcoma.

Patients with metastatic osteogenic sarcoma included those who had relapsed on adjuvant chemotherapy and those presenting with overt metastatic disease at the time of diagnosis. The multidisciplinary programme incorporated the V-MTX-CF regimen administered weekly, which is significantly more effective than the tri-weekly schedule. ${ }^{2}$ Surgery and radiotherapy and extended chemotherapy were integrated into the programme.

\section{Patients and methods}

Twenty-five consecutive patients referred to the Sidney Farber Cancer Institute and the Children's Hospital Medical Centre from

\footnotetext{
Sidney Farber Cancer Institute, Harvard Medical School, Boston Massachusetts

NORMAN JAFFE, MB, DIP PAED, chief of paediatric solid tumour service DEMETRIUS TRAGGIS, MD, senior associate

EMIL FREI, MD, professor and director of institute

Children's Hospital Medical Centre and Joint Centre for Radiation Therapy, Boston, Massachusetts

J ROBERT CASSADY, MD, radiation therapist

ROBERT M FILLER, MD, clinical chief, department of surgery

HUGH WATTS, MD, orthopaedist
}

March 1974 to December 1975 were entered into the study. Four patients refused treatment. Of the 21 evaluable patients $20 \mathrm{had}$ classical osteogenic sarcoma and one had chondrosarcoma. Nineteen patients had radiographically evident pulmonary metastases, one had iliac node metastases, and two had bone metastases. Three of the patients had pulmonary metastases at the time of initial diagnosis and 18 had relapsed from adjuvant chemotherapy. Chemotherapy had comprised adriamycin only in five patients, V-MTX-CF only in three, and both adriamycin and V-MTX-CF in 10. Thirteen of these patients had received adjuvant chemotherapy here and five had been treated elsewhere before referral. The age range of the 21 patients was 6 to 19 years. Twelve of the 21 patients were male.

\section{PRINCIPLES OF TREATMENT}

Since there is evidence that chemotherapy provides definitive treatment for micrometastases in the initial adjuvant programme, every effort was made to achieve a complete remission. After remission had been achieved chemotherapy was continued in an effort to eradicate micrometastases. The multidisciplinary regimen designed to achieve complete remission was adjusted depending on the mode of presentation, extent of disease, and previous treatment (see table I). In all circumstances, the senior representatives of the disciplines (surgery, radiation therapy, and paediatric oncology) met when the patient was admitted into the study and the programme was planned for the individual patient in keeping with the above principles.

The patients fell into four treatment sequence categories, as indicated in table I. The interval between adjuvant treatment and the end of specific treatment was never longer than three weeks. In five patients pulmonary metastases were completely removed by surgery. One patient was treated with adriamycin. V-MTX-CF was given in weekly courses for four to eight weeks to 15 patients (CHEM I). In three of these patients complete tumour regression occurred. In the remaining 12 there was either no response or partial response and surgery was used when feasible to create a complete remission. In 10 patients secondary treatment with four courses of weekly V-MTX-CF (CHEM II) combined with whole-lung radiotherapy (see below) was used. After this treatment V-MTX-CF was administered every two to three weeks for 18 months (CHEM III). Two of these patients received concurrent adriamycin.

The weekly V-MTX-CF regimen is shown in fig 1 . Courses of treatment were administered every seven days, and the dose of methotrexate was increased from $3 \mathrm{~g} / \mathrm{m}^{2}$ to $6 \mathrm{~g} / \mathrm{m}^{2}$ and $7.5 \mathrm{~g} / \mathrm{m}^{2}$ at weekly intervals. A minimum of four courses were administered before treatment was considered ineffective, and treatment was continued to a maximum of eight courses provided there was some response.

Before each course of treatment creatinine clearances were measured, and serum creatinine was measured at 24-hour intervals during each course. MTX levels were measured daily. If a level over $100 \mathrm{nmol} / \mathrm{l}$ $(4.5 \mu \mathrm{g} / 100 \mathrm{ml})$ at 72 hours was detected citrovorum factor was continued until a result below this level was obtained. Leucocyte and platelet counts were measured and liver function studies performed twice a week. Chest radiographs were obtained every two to three weeks, before each course of treatment. Recently, radionucleotide bone scanning was adopted as an additional investigation to be performed every six to 12 months.

After the maximum possible surgical removal of known tumour patients who had pulmonary metastases received whole-lung irradiation (1500 rads/10 treatments/12 elapsed days) concurrently with four weekly courses of V-MTX-CF. When limited residual disease remained or margins of resections were uncertain after surgery additional irradiation (1500 to 2500 rads) was delivered to localised areas of one or both lungs.

One patient with iliac node metastases incompletely removed by surgery received 5000 rads/25 treatments $/ 52$ elapsed days, while another patient received $4041 \mathrm{rads} / 20$ treatments/33 elapsed days to the tumour bed after removal of a rib metastasis. The approach was 
TABLE I-Treatment strategy, categories, and results

\begin{tabular}{|c|c|c|c|}
\hline Treatment sequence & $\begin{array}{c}\text { No of } \\
\text { patients }\end{array}$ & $\begin{array}{l}\text { Complete remission } \\
\text { achieved }\end{array}$ & $\begin{array}{l}\text { Complete remission } \\
\text { continues }\end{array}$ \\
\hline $\begin{array}{l}\text { Surgery } \rightarrow \text { (CHEM II + radiotherapy) } \rightarrow \text { CHEM III } \\
\text { CHEM I } \rightarrow \text { Surgery } \rightarrow \text { CHEM III } \\
\text { CHEM I } \rightarrow \text { Surgery } \rightarrow \text { (CHEM II + radiotherapy) } \rightarrow \text { CHEM III } \\
\text { CHEM I } \rightarrow \text { CHEM III } \\
\text { Adriamycin only }\end{array}$ & $\begin{array}{l}5 \\
3 \\
5 \\
7 \\
1\end{array}$ & $\begin{array}{l}5 \\
3 \\
4 \\
3 \\
0\end{array}$ & $\begin{array}{l}5 \\
2 \\
4 \\
3 \\
0\end{array}$ \\
\hline Total & 21 & 15 & 14 \\
\hline
\end{tabular}

CHEM I: V-MTX-CF weekly for 4-8 weeks. CHEM II: V-MTX-CF weekly for 4 weeks. CHEM III: V-MTX-CF every two to three weeks for 18 months. Two received concurrent adriamycin.

6 hours

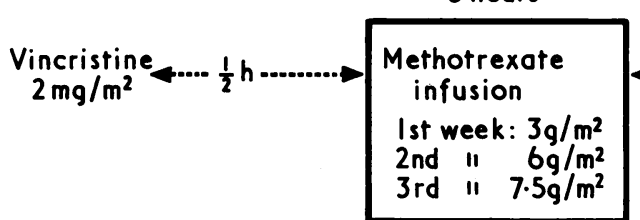

24 hours

48 hours

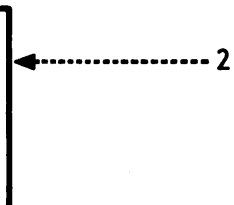

Oral citrovorum factor $15 \mathrm{mg} / 6 \mathrm{~h}$

FIG 1-Weekly V-MTX-CF regimen. First oral dose of citrovorum factor is given three hours after last intravenous dose.

based on the suggested evidence for potentiation between V-MTX-CF and radiation treatment. ${ }^{9} \mathrm{~A} 4$ - or $8-\mathrm{MeV}$ linear accelerator was used.

To provide a frame of reference for interpreting the results a historical control group was used. This included 82 consecutive patients who developed pulmonary or other metastases at this centre before we started adjuvant studies (March 1972). The survival of this historical control group was plotted from the time metastatic disease was detected until death.

Life table plots were computed according to the method of Cutler ${ }^{10}$ and were analysed by the Wilcoxon test as modified for life table data by Gehan. ${ }^{11}$

\section{Results}

Fifteen of the 21 patients received weekly V-MTX-CF as initial treatment, and within this group it was possible to determine the effectiveness of such treatment alone in producing tumour regression (table II). Seven of the 15 patients had complete or partial responses $(48 \%)$. Response was substantially influenced by whether the patients had previously received V-MTX-CF on a three-week schedule. Thus of nine patients who had received such treatment, three partially responded, while of the six patients who had not received V-MTX-CF four responded, three of them completely.

The survival of the study group and the historical control group are shown in fig 2 . The median survival of the historical control group was nine months, and only two patients remained alive at 30 months. Survival among the 21 study patients was significantly better by both the double Wilcoxon and $\chi^{2}$ analysis $(P=0.001)$. More important, however, was the fact that 14 of the 15 patients rendered clinically free of disease continued to be free from over two to over 18 months. This included one patient with chondrosarcoma who achieved a complete remission initially by bilateral thoracotomy.

\section{TOXICITY}

Five per cent of the courses of V-MTX-CF were associated with mucositis or myelosuppression, or both. There were no deaths and no

TABLE II-Effect of weekly $V-M T X-C F$ in relation to previous treatment

\begin{tabular}{l|c|c|c|c}
\hline Previous chemotherapy & $\begin{array}{c}\text { No of } \\
\text { patients }\end{array}$ & $\begin{array}{c}\text { Complete } \\
\text { remission }\end{array}$ & $\begin{array}{c}\text { Partial } \\
\text { remission }\end{array}$ & $\begin{array}{c}\text { No } \\
\text { remission }\end{array}$ \\
\hline $\begin{array}{c}\text { V-MTX-CF every 3 weeks } \\
\text { V-MTX-CF every threeweeks + adriamycin }\end{array}$ & 6 & & 3 & 3 \\
Adriamycin & 4 & 1 & 1 & 2 \\
None & 2 & 2 & & \\
\hline Total* & 15 & 3 & 4 & 8 \\
\hline
\end{tabular}

* Six patients not included: five achieved complete remission on surgery and one was not treated with weekly methotrexate. infection or haemorrhage due to chemotherapeutically-induced myelosuppression. Nausea and vomiting during the methotrexate infusion was observed in $30-40 \%$ of courses. This varied in severity, but in no case did treatment have to be discontinued. On the weekly V-MTX-CF programme, anorexia associated with some degree of weight loss occurred in most patients. Anorexia decreased substantially and weight loss was either stabilised or regained when patients progressed to the CHEM II schedule.

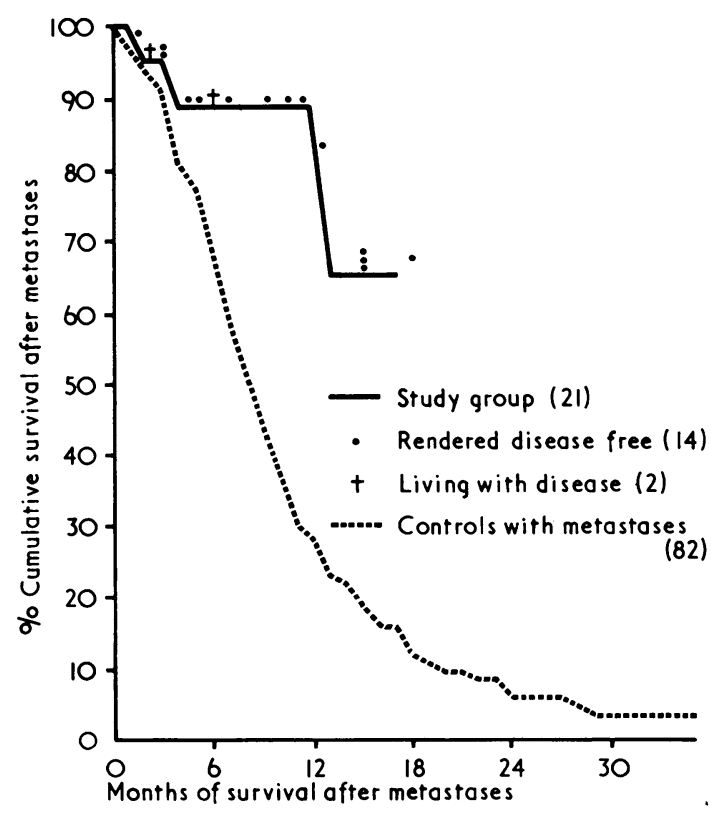

FIG 2-Survival in 21 patients with metastases after multidisciplinary treatment compared with survival in 82 patients with metastases previously treated at the Sidney Farber Cancer Institute. Five patients in the study group died.

Pulmonary metastases were detected in one patient after treatment with radiation therapy and V-MTX-CF. In retrospect these had been present before the combined treatment. They were removed by thoracotomy and showed central areas of necrosis.

Information on the surgery will be described separately. There were no postoperative complications and there was no evidence that the chemotherapy interfered with immediate wound healing. Pneumonitis and fibrosis attributable to radiotherapy were not observed. Transient non-progressive abnormalities in liver function associated with V-MTX-CF occurred. 


\section{Discussion}

We have described a "curative intent" approach to patients with macrometastatic osteogenic sarcoma. There is increasing evidence that chemotherapy provides long-term control and perhaps eradicates micrometastases in a substantial proportion of patients with osteogenic sarcoma in whom the primary lesion is controlled. ${ }^{3-5}{ }^{12}$ It is reasonable, therefore, to use this approach against micrometastases in patients with overt metastatic disease that can be removed or destroyed by a multidisciplinary approach. This was, in fact, achieved in 15 of the 21 patients. While the follow-up was not long enough to determine whether our approach has been successful, it shows promise, considering that 14 of the 15 patients remain clinically free of disease.

Chemotherapy produced complete remission in only three patients, and surgery was required in the remaining patients. In an earlier study, before the development of effective chemotherapy for osteogenic sarcoma, surgery for removal of pulmonary metastases produced a $27 \%$ five-year survival rate in 29 selected patients. ${ }^{13}$ This approach should not be limited to patients with pulmonary metastatic disease. In our study one patient with iliac node metastases and two with skeletal lesions achieved complete remission with surgery. Surgery included a rib and femoral neck resection in one patient and resection of the lower femur in the second. Internal prostheses were used in both patients.

The chemotherapy included initial weekly V-MTX-CF. This programme has produced objective responses in seven out of eight patients with overt clinical disease who had not received V-MTX-CF. Four of the patients had primary lesions and three had pulmonary metastases. ${ }^{2}$ In this study four out of six patients who had not received previous V-MTX-CF responded to the weekly programme, three of them completely. The weekly programme was substantially less effective in patients who had received V-MTX-CF in three-week courses. Nevertheless, partial responses were achieved in three out of nine patients, which is comparable to results achieved with adriamycin or with the tri-weekly V-MTX-CF programme. ${ }^{72} \mathrm{With}$ appropriate attention to urine volume and with careful pharmacological and toxicological monitoring, unacceptable toxicity was not observed.

While osteogenic sarcoma is not considered to be a radiosensitive tumour, radiotherapy has reduced, though not eradicated, primary lesions. ${ }^{14}$ It is reasonable to postulate that radiation treatment might be effective in helping to eradicate microscopic disease. The use of radiotherapy and chemotherapy concurrently was suggested by the observation that V-MTX-CF produced recall erythema in a previously irradiated area similar to that produced by actinomycin D. ${ }^{9}$ Radiation pneumonitis and fibrosis were not observed, though the period of observation may not have been long enough. The dose of irradiation (1500 rads in 12 days) was similar to that used for whole-lung treatment in children with Wilms's tumour. ${ }^{15}$

In all patients but one pulmonary lesions removed surgically showed osteogenic sarcoma pathologically. In one patient chemotherapy was started and tumours that were originally growing rapidly stabilised. Chemotherapy, therefore, was continued for two years. Radiographically, the lesions initially appeared "soft" but became progressively denser over the next 12 months. After a six-month course of adriamycin $\left(75 \mathrm{mg} / \mathrm{m}^{2}\right.$ every three weeks) biopsy specimens of the lesions were obtained. These showed calcified osteoid without morphological evidence of osteogenic sarcoma. Possibly this phenomenon will be seen with increasing frequency with improved treatment and survival in patients with osteogenic sarcoma. Certainly, lack of regression in metastatic lesions does not mean that the treatment is ineffective and should be discontinued.

The historical control group from this centre helped us to interpret our findings. Our impression that there were far fewer pulmonary metastases in the study group than in the control group is currently being analysed. Since most of the study patients had previously received and failed on adjuvant treatment, we have postulated that such treatment reduced but did not eradicate micrometastases, and these patients might therefore be expected to have fewer overt pulmonary metastases than the controls.

We hope this and comparable studies in osteogenic sarcoma will repeat the precedent set in Wilms's tumour. Initially it was shown that actinomycin $\mathrm{D}$ could increase the cure rate in patients in whom the primary lesion was controlled by local treatment. ${ }^{16}$ Then it was shown that even in patients with overt metastatic disease control of bulk disease by radiotherapy and of microscopic disease by chemotherapy could result in long-term tumour-free survival. ${ }^{16}$ These examples may be generally applicable to cancer treatment. For example, it has been shown that adjuvant chemotherapy may control microscopic metastases in patients with primary breast cancer. ${ }^{17} 18$ This multidisciplinary approach to producing a complete remission and then giving chemotherapy to eradicate micrometastic disease is currently being assessed in patients with early metastatic breast cancer.

This work was supported in part by a research grant (CA06516) from the National Cancer Institute and by a grant (RR-05526) from the Division of Research Facilities and Resources. National Institutes of Health.

Requests for reprints should be addressed to Dr Norman Jaffe, Sidney Farber Cancer Institute, 44 Binney Street, Boston, Massachusetts 02115 .

\section{References}

1 Rosen, G, et al, Cancer, 1976, 37, 1.

2 Jaffe, $\mathrm{N}$, et al, Cancer, in press.

$3 \mathrm{Jaffe}, \mathrm{N}$, et al, New England fournal of Medicine, 1974, 291, 1974.

4 Rosen, G, et al, Proceedings of the American Society of Clinical Oncology, $1975,16,227$.

5 Sutow, W W, Sullivan, M P, and Fernbach, D J, Proceedings of the American Association for Cancer Research, 1974, 15, 20.

6 Pratt, C B, Hustu, H O, and Shanks, E, Proceedings of the American Association for Cancer Research, 1974, 15, 19.

$7 \mathrm{Jaffe}, \mathrm{N}$, et al, Cancer, 1973, 31, 1367.

8 Rosen, G, et al, Cancer, 1974, 33, 1151.

${ }^{9}$ Rosen, G, et al, Cancer, 1975, 35, 622.

10 Cutler, S J, and Ederer, F J, Chronic Diseases, 1958, 8, 699.

11 Gehan, E A, Biometrika, 1965, 52, 203.

12 Cortes, E P, et al, fournal of the American Medical Association, 1972, 221, 1132.

13 Martini, et al, Annals of Thoracic Surgery, 1971, 12, 271.

14 Cade, S, Fournal of Royal College of Surgeons of Edinburgh, 1955, 1, 79.

15 Cassady, J R, et al, Cancer, 1973, 32, 598.

${ }_{16}$ Farber, S, Fournal of the American Medical Association, 1966, 198, 826.

17 Bonadonna, G, et al, New England fournal of Medicine, 1976, 294, 406.

18 Fisher, B, et al, New England fournal of Medicine, 1975, 292, 118. 